\section{Defective Somatosensory Inhibition and Plasticity Are Not Required to Develop Dystonia}

\author{
Anna Latorre, MD, PhD, ${ }^{1 *}$ (D) Antoniangela Cocco, MD, ${ }^{2,3}$ \\ Kailash P. Bhatia, FRCP, MD, ${ }^{1}$ (D) \\ Roberto Erro, MD, PhD, ${ }^{4}$ (D) Elena Antelmi, MD, PhD, ${ }^{5}$ \\ Antonella Conte, MD, PhD, ${ }^{6,7}$ John C. Rothwell, PhD, ${ }^{1}$ (D) \\ and Lorenzo Rocchi, MD, $\mathrm{PhD}^{1}$ (D)
}

\begin{abstract}
${ }^{1}$ Department of Clinical and Movement Neurosciences, Queen Square Institute of Neurology, University College London, London, United Kingdom ${ }^{2}$ Department of Neurology, IRCCS Humanitas Research Hospital, Milan, Italy ${ }^{3}$ Department of Neuroscience, Catholic University, Milan, Italy ${ }^{4}$ Department of Medicine, Surgery and Dentistry "Scuola Medica Salernitana", University of Salerno, Baronissi, Italy ${ }^{5}$ Neurology Unit, Movement Disorders Division, Department of Neurosciences, Biomedicine and Movement Sciences, University of Verona, Verona, Italy ${ }^{6}$ Department of Human Neurosciences, Sapienza, University of Rome, Rome, Italy ${ }^{7}$ IRCCS Neuromed, Pozzilli, Italy
\end{abstract}

ABSTRACT: Background: Dystonia may have different neuroanatomical substrates and pathophysiology. This is supported by studies on the motor system showing, for instance, that plasticity is abnormal in idiopathic dystonia, but not in dystonia secondary to basal ganglia lesions.

Objective: The aim of this study was to test whether somatosensory inhibition and plasticity abnormalities reported in patients with idiopathic dystonia also occur in patients with dystonia caused by basal ganglia damage.

Methods: Ten patients with acquired dystonia as a result of basal ganglia lesions and 12 healthy control subjects were recruited. They underwent electrophysiological testing at baseline and after a single 45-minute session of high-frequency repetitive somatosensory stimulation. Electrophysiological testing consisted of somatosensory temporal discrimination, somatosensory-evoked potentials (including measurement of early and late high-frequency oscillations and the spatial inhibition ratio of $\mathrm{N} 20 / 25$ and P14 components), the recovery cycle of paired-pulse

${ }^{*}$ Correspondence to: Dr. Anna Latorre, Department of Clinical and Movement Neurosciences, UCL Queen Square Institute of Neurology, Queen Square, London WC1N 3BG, United Kingdom; E-mail: a.latorre@ucl.ac.uk

Received: 19 August 2020; Revised: 21 October 2020; Accepted: 18 November 2020

Published online in Wiley Online Library

(wileyonlinelibrary.com). DOI: 10.1002/mds.28427 somatosensory-evoked potentials, and primary motor cortex short-interval intracortical inhibition.

Results: Unlike previous reports of patients with idiopathic dystonia, patients with acquired dystonia did not differ from healthy control subjects in any of the electrophysiological measures either before or after high-frequency repetitive somatosensory stimulation, except for short-interval intracortical inhibition, which was reduced at baseline in patients compared to control subjects.

Conclusions: The data show that reduced somatosensory inhibition and enhanced cortical plasticity are not required for the clinical expression of dystonia, and that the abnormalities reported in idiopathic dystonia are not necessarily linked to basal ganglia damage. (C) 2020 International Parkinson and Movement Disorder Society

Key Words: dystonia; inhibition; plasticity; somatosensory system; temporal discrimination

Dystonia is a heterogeneous disorder with variable distribution (from focal to generalized), phenomenology (isolated or combined to additional signs), and etiology (inherited, acquired, or idiopathic). ${ }^{1}$ Treatment strategies and their outcomes also differ between dystonia subtypes. $^{2-4}$ Such features are consistent with the concept that different dystonias may have different neuroanatomical substrates ${ }^{5}$ and pathophysiology. ${ }^{6}$ Evidence for the latter comes from studies in the motor system. Although some physiological features are common to many types of dystonia, such as reduced intracortical inhibition in motor cortex, others, such as motor cortex plasticity, are not. ${ }^{7-12}$ Plasticity is abnormal in idiopathic dystonia ${ }^{10,13}$ but does not seem to be required for the clinical expression of dystonia secondary to basal ganglia lesions, ${ }^{10}$ even though dystonia normally develops months after the brain insult, suggesting an underlying plastic reorganization.

Although clinical symptoms of dystonia relate to disorders of movement, there are clear abnormalities in the somatosensory system, at least in idiopathic dystonia. One of the most consistent findings is an increased threshold for somatosensory temporal discrimination (STDT). ${ }^{14,15}$ Physiologically, this is associated with reduced excitability of the inhibitory circuits in the primary somatosensory cortex (S1) that are required to heighten temporal acuity, ${ }^{16,17}$ as reflected by reduced inhibition of pairedpulse somatosensory-evoked potentials (PP-SEPs) and decreased area of high-frequency oscillations (HFOs). ${ }^{18}$ Patients with idiopathic dystonia also show abnormal 
inhibition in the spatial domain, as demonstrated by defective suppression of SEPs obtained by simultaneous stimulation of the median and ulnar nerves ${ }^{19}$ and of contiguous fingers. ${ }^{18}$ In healthy volunteers, STDT and S1 inhibition are improved by a period of high-frequency repetitive somatosensory stimulation (HF-RSS), whereas it has the opposite effect in idiopathic dystonia. ${ }^{20}$ This has been attributed to abnormal somatosensory plasticity akin to that observed in the motor system.

In this article, we tested whether the abnormalities of STDT, S1 inhibition, and S1 plasticity that are seen in patients with idiopathic dystonia also occur in patients with dystonia acquired after basal ganglia damage. In addition, to evaluate the interaction between somatosensory and motor systems, we also investigated the inhibitory circuitry within M1 before and after HF-RSS. Our hypothesis was that the findings in secondary dystonia (SD) would differ from those previously reported in idiopathic dystonia, providing further evidence that dystonia can arise from different, probably independent, mechanisms.

\section{Subjects and Methods}

Ten patients affected by acquired dystonia secondary to structural brain lesions (here named SD) were consecutively recruited from the National Hospital for Neurology and Neurosurgery, Queen Square, London, United Kingdom. Inclusion criteria comprised: (1) unilateral distribution of dystonia, with clear involvement of the upper limb; (2) no significant pyramidal signs or sensory abnormalities of the limbs affected by dystonia; and (3) structural brain lesion involving the basal ganglia, contralateral to the clinically affected side, confirmed by brain MRI. All patients were assessed at least 3 months after their last set of botulinum toxin injections and off medications that act on the central nervous system (such as trihexyphenidyl or clonazepam). Disease severity was assessed with the Unified Dystonia Rating Scale. ${ }^{21}$ Clinical features are summarized in Supporting Information Table S1. A control group of 12 age-matched, healthy control subjects (HCs) was also enrolled in the study. All experimental procedures were performed as previously reported in the literature. ${ }^{16,18,20,22}$ In summary, all participants underwent electrophysiological and behavioral testing at baseline (T0) and after (T1) a single 45-minute session of HFRSS, which consists of $20-\mathrm{Hz}$ trains of square wave electrical pulses of $200 \mu \mathrm{s}$ duration delivered for 1 second, with 5 -second intertrain intervals, applied on the tip of the index finger [test finger (TF)] of the dystonic hand and of the right hand in HCs (test hand). Measures collected included: (1) STDT, tested on the TF and three control fingers (thumb of the test hand and thumb and index finger of the contralateral hand, named, respectively, F1, F2, and F3); (2) several variables derived from SEPs obtained by stimulation of TF and F1, that is, N20/P25 and P14 latencies and amplitudes, area of early and late parts of HFOs (e$\mathrm{HFO}$ and 1-HFO, respectively), recovery cycle of PPSEPs, and spatial inhibition ratio (SIR) of N20/25 (Q20) and P14 (Q14); and (3) short-interval intracortical inhibition (SICI), measured from the abductor pollicis brevis and abductor digiti minimi muscles, and obtained with a test motor-evoked potential of approximately $1 \mathrm{mV}$. The methods used are detailed in the Supporting Information. The experimental protocol was approved by the local institutional review board and conducted in accordance with the Declaration of Helsinki after each participant signed a written consent form.

Details about statistical analysis are given in the Supporting Information. In brief, age, HF-RSS threshold and stimulation intensity, SEP intensity for TF and F1, resting motor threshold, and $1 \mathrm{mV}$ intensity were compared between patients with SD and HCs by means of unpaired $t$ tests. Sex was compared with the Fisher's exact test. Mixed analyses of variance were used to compare other variables across the two groups (STDT intensities and values, SEP N20/P25 and P14 latencies and amplitudes, Q20, Q14, area of e-HFO and l-HFO, N20/P25 and P14 recovery cycle, SICI).

\section{Results}

There were no differences in age, sex, transcranial magnetic stimulation (TMS) thresholds, and electrical/ magnetic stimulation intensities between groups (Supporting Information Table S2). Unlike the typically elevated STDT in idiopathic dystonia, there was no significant difference in STDT values between HCs and patients. HF-RSS had no effect on the stimulation intensity used for STDT, N20, and P14 latencies and test motor-evoked potentials (raw values for these variables are listed in Supporting Information Table S3). Main effects and interactions of all analyses of variance are listed in Supporting Information Table S4.

Significant results are shown in Figures 1 and 2 . Except from SICI, all baseline values were not statistically different between patients and HCs.

Overall, there were no significant differences in the effects of HF-RSS in HCs and patients with SD. In both groups, HF-RSS increased the amplitude of the subcortical P14 and cortical N20/P25 components of the SEP and decreased (i.e., improved) STDT in the TF, but not F1 (all $P<0.01$ ) (Fig. 1A-C). Baseline SICI was less effective in patients with SD than in HCs but was enhanced in the abductor pollicis brevis muscle to the same degree in both groups after HF-RSS and for all conditioning stimulus intensities (all $P<0.05)$. There was no change in SICI in the distant abductor digiti minimi muscle. 

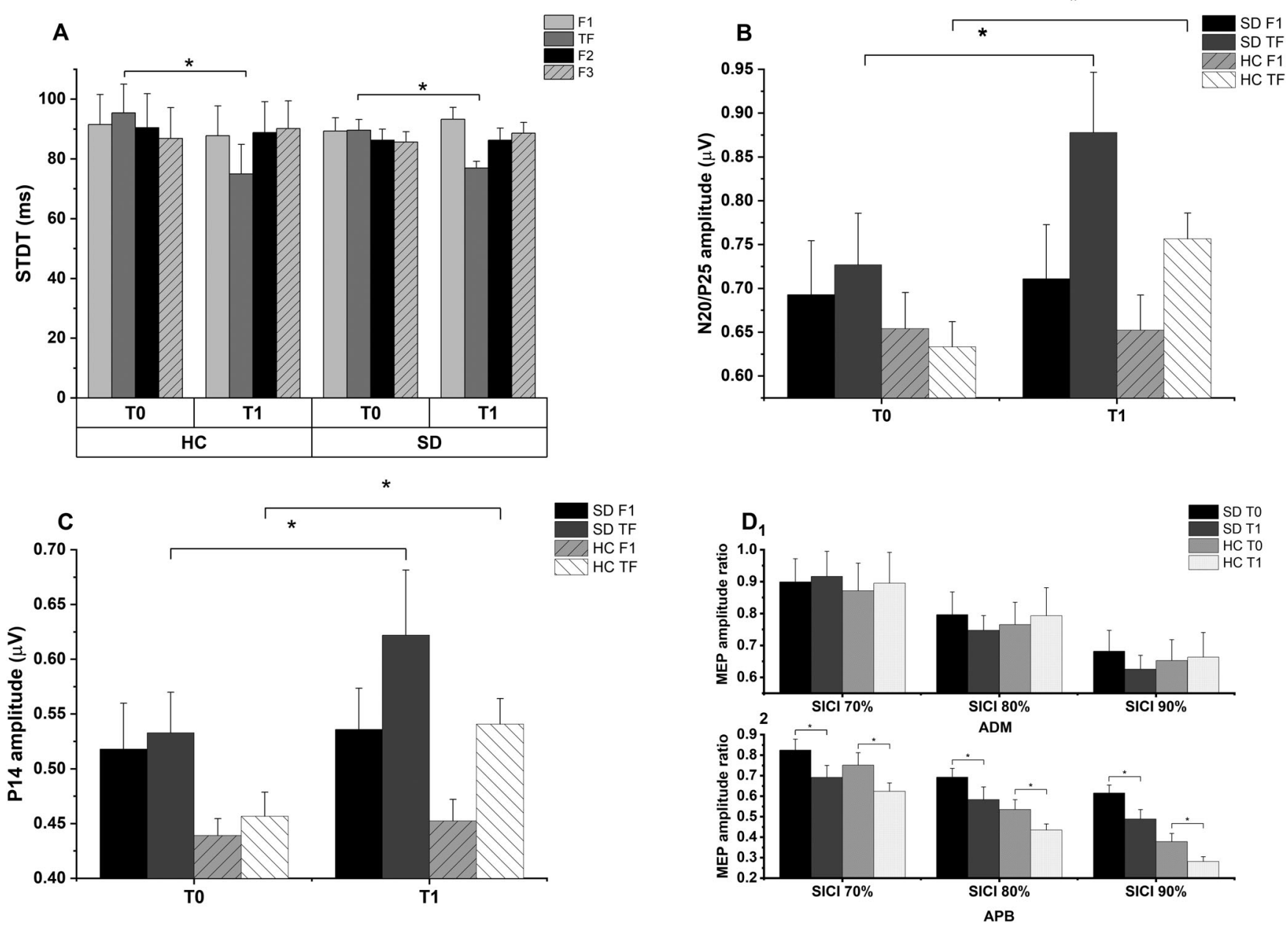

FIG 1. (A) STDT values obtained from the thumb (F1) and the index finger [test finger (TF)] of the test hand, and thumb (F2) and index finger (F3) of the contralateral hand, before (TO) and immediately after (T1) high-frequency repetitive somatosensory stimulation (HF-RSS) was applied on the TF, in healthy control subjects (HCs) and patients with secondary dystonia (SD). HF-RSS produced a significant decrease of STDT in TF only ( $P<0.01)$. (B, C) Amplitude of N20/P25 (B) and P14 (C) components of somatosensory-evoked potentials obtained by stimulation of the thumb (F1) and the index finger (TF) of the test hand in HCs and patients with SD, before (T0) and after (T1) HF-RSS applied on the TF. HF-RSS induced a significant increase of N20/P25 amplitude $(P<0.01$ in both cases), as well as an increase in P14 (both $P<0.01$ ). (D) Effect of HF-RSS on SICl on abductor pollicis brevis (APB) and abductor digiti minimi (ADM) at different intensities of the conditioning TMS stimulus (CS) (70\%, $80 \%$, and $90 \%$ of active motor threshold (AMT)), in HCs and patients with SD, at T0 and T1. HF-RSS induced an increase in SICl, irrespective of the strength of the conditioning TMS pulse, in the APB, but not in the ADM (all $P<0.05$ ). Asterisks indicate statistical significance. Error bars indicate standard error. MEP, motor-evoked potential.

Figure 2 shows that the effectiveness of somatosensory intracortical inhibition was enhanced after HF-RSS in both groups, as indicated by the increase in e-HFO and 1 -HFO areas $(P<0.01)$, and the increased amount of inhibition in the N20/P25 and P14 PP-SEP recovery cycle, in both groups and at all interstimulus intervals (all $P<0.01$ ). SIR was also improved in HCs and patients with $\mathrm{SD}$, as indicated by the decrease in Q14 and Q20 (both $P<0.01$ ).

\section{Discussion}

The main finding of this study is that patients with SD caused by lesions in the basal ganglia did not differ from HCs in any of the electrophysiological variables tested either before or after HF-RSS, except for SICI, which was reduced at baseline in patients with SD compared with HCs. This means that STDT and somatosensory inhibition, investigated by PP-SEP, SIR, and HFOs, as well as cortical plasticity induced by HF-RSS, are normal in SD, whereas motor inhibitory mechanisms are defective, as previously described. ${ }^{10}$

This is the first study to report that STDT is normal in SD. STDT reflects the ability to discriminate two consecutive cutaneous electrical stimuli; it relies on inhibitory mechanisms within $S 1^{17,18,23,24}$ and on functional integrity of the basal ganglia. ${ }^{25}$ Increased STDT is the most consistent behavioral abnormality observed in idiopathic dystonia. ${ }^{18,20,26-28}$ Indeed, it has been 

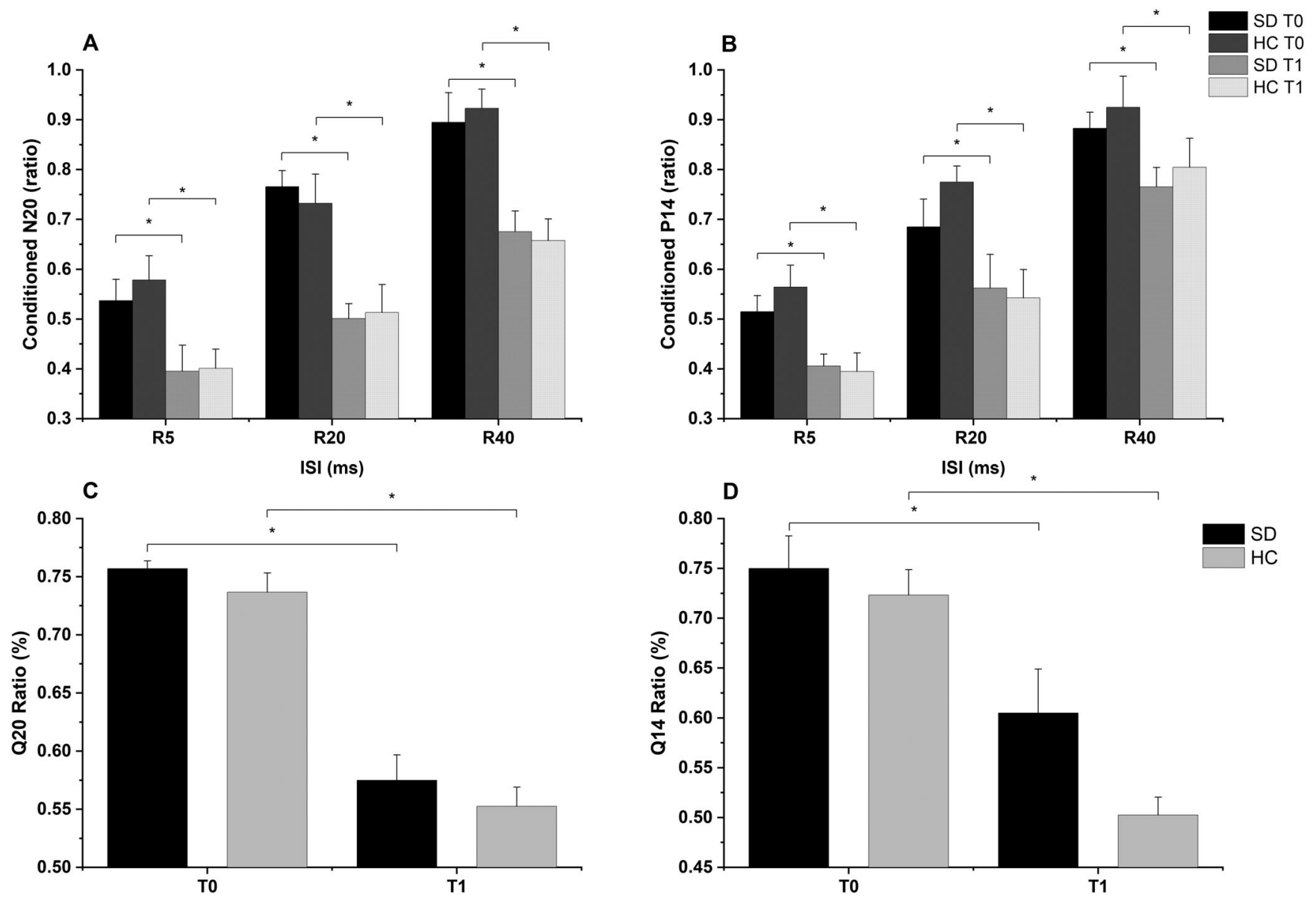

FIG 2. Recovery cycle of N20/P25 (A) and P14 (B) components of somatosensory-evoked potentials at interstimulus intervals (ISIs) of 5 , 20 , and 40 ms before (TO) and immediately after (T1) high-frequency repetitive somatosensory stimulation (HF-RSS) applied on the test finger (TF). HF-RSS significantly increased the amount of inhibition on both the N20/P25 (all $P<0.001$ ) and P14 (all $P<0.01$ ) components, in both groups and at all ISls. Depicted in the lower row is the spatial inhibition ratio of N20/P25 (Q20) (C) and P14 (Q14) (D), before (T0) and after (T1) HF-RSS, in healthy control subjects and patients with secondary dystonia (SD). HF-RSS increased the spatial inhibition ratio in both groups by decreasing the Q20 (both $P<0.01$ ) and the Q14 (both $P<0.01$ ). Asterisks indicate statistical significance. Error bars indicate standard error.

proposed to be an endophenotypic trait of dystonia ${ }^{25,29}$ because of its lack of correlation with disease manifestation and treatment. ${ }^{30-32}$ In view of this evidence, the finding of normal STDT in SD indicates that, although all our patients displayed basal ganglia lesions, this is not sufficient to affect temporal discrimination..$^{25,27}$ In addition, increased STDT in idiopathic dystonia is associated with reduced excitability of inhibitory circuits in $\mathrm{S} 1,{ }^{18,20,31,33}$ whereas in this study, S1 inhibitory excitability was normal in patients with SD.

The measures of somatosensory inhibition applied in this study have never been investigated in SD, other than in SD caused by lesions in the somatosensory pathways. $^{34,35}$ None of our patients presented with lesions involving the somatosensory system, which was functionally intact. However, the results differed from those in idiopathic dystonia, in which there is reduced PP-SEP suppression, ${ }^{20,23}$ impaired SIR, ${ }^{18-20}$ and reduced HFO. ${ }^{18,20}$ This implies that, although the loss of somatosensory inhibition has a role in the pathophysiology of idiopathic dystonia, it is of less relevance in patients affected by SD as a result of basal ganglia lesions.

In contrast with the preserved inhibition in the somatosensory system, we found that, as reported by others, M1 SICI is reduced in SD. ${ }^{10,36}$ Reduced SICI is considered a hallmark feature of dystonia, although this notion has been recently questioned and its pathophysiological significance is still not clear. ${ }^{37}$ In this case, reduced SICI, which is mediated by M1 inhibitory interneurons, might be a direct consequence of deranged basal ganglia output to M1, because of structural lesions; however, the causal relationship between abnormal SICI and dystonia is difficult to ascertain. Indeed, SICI can be reduced also in other basal ganglia diseases, ${ }^{38,39}$ in functional dystonia, ${ }^{11,12}$ and in unaffected DYT1 gene mutations carriers. ${ }^{9}$ It is therefore suspected that other abnormalities, together with the lack of inhibition, are required for dystonia to manifest. ${ }^{37}$ 
Disordered control of plasticity has been a frequent finding in M1 and also, more recently, in S1 of patients with idiopathic dystonia. ${ }^{10,13,20,40}$ However, our present results in S1 indicate that, as previously reported in $\mathrm{M} 1,{ }^{10}$ plasticity may be normal in SD. Thus, HF-RSS induced the same amount of plasticity as controls: it led to a short-term increase in the excitability of inhibitory circuitry within S1 and M1, which was similar to that in HCs. ${ }^{16}$ This result is in striking contrast with the opposite effect of HF-RSS on S1 inhibition in idiopathic dystonia, ${ }^{20}$ and there are a number of possible explanations for it. First, in our previous study, ${ }^{20}$ HF-RSS was applied over a body part not affected by dystonia. However, this would be unlikely to account for the normal STDT and homeostatic plasticity found in SD, because similar abnormalities are seen when testing body parts uninvolved in dystonia. ${ }^{3,41}$ Second, our patients with SD were affected by hemidystonia and not focal dystonia, and it is possible that their pathophysiological mechanisms are different. ${ }^{6}$ Indeed, there is growing evidence that the causative mechanisms of dystonia might differ according to the subtypes (for instance, generalized versus focal), but also according to the presence of specific clinical features such as tremor or task specificity. ${ }^{42}$ Finally, and more importantly, in idiopathic dystonia, HF-RSS acted on top of a defective somatosensory system (as a result of lack of inhibition), and this could contribute to the paradoxical response. Whatever the explanation, the data show that abnormally enhanced cortical plasticity is not required for the clinical expression of all types of dystonia, and that it is not necessarily linked to basal ganglia damage.

Linking basal ganglia dysfunction and somatosensory abnormalities in dystonia is a conundrum yet to be resolved. One line of evidence of this connection comes from the findings that STDT relies on a complex network of brain structures, including the basal ganglia. ${ }^{28,43}$ The neuroanatomical basis of this interaction is not clear, but one possibility is represented by direct connections between the striatum and the somatosensory cortex. ${ }^{33}$ However, other lines of evidence might suggest that alterations in the basal ganglia and somatosensory function in dystonia are relatively independent. In patients with idiopathic cervical dystonia, Antelmi and coworkers ${ }^{18}$ found that electrophysiological markers of S1 inhibition and dystonia were independently associated with abnormal STDT, leading to speculation that other factors beyond alterations in S1 computation might contribute to increased STDT, such as basal ganglia dysfunction. This would be in line with the current results, which suggest that structural damage in the basal ganglia does not necessarily lead to somatosensory dysfunction. However, given the existent controversy, we acknowledge that both interpretations are possible.
Do our results have an impact on the clinical management of patients with SD? The most obvious clinical evidence of the sensory system involvement in dystonia is the sensory trick, that is, the relief of dystonic activity by tactile stimulation of the affected body part. Although the sensory trick prevalence is variable in idiopathic dystonia, ${ }^{44,45}$ it is certainly higher compared with SD. ${ }^{46}$ The mechanisms underlying sensory trick are not well understood, but it has been proposed that tactile stimulation provides additional input to the brain that normalizes a preexisting abnormal gating of sensory input to motor circuits. ${ }^{47}$ This might be one reason why the sensory trick is more common in idiopathic dystonia, where abnormal somatosensory processing has been demonstrated, ${ }^{20}$ than in SD, in which sensory processing may be normal, as suggested by the present findings. It has been proposed that modulation of somatosensory deficit via repetitive peripheral stimulation might be a suitable treatment option for idiopathic dystonia ${ }^{20}$; however, because somatosensory inhibition and plasticity are not affected in SD, addressing these abnormalities in SD would probably not be justified.

The present results should, however, be considered in light of some limitations, including the small sample size and the heterogeneity of the patients' group, because of the variable degree of dystonia and different involvement of the basal ganglia (Supporting Information Table S1).

Although classically considered a basal ganglia disorder, dystonia has more recently been framed as a network dysfunction, involving the thalamus, cerebellum, and sensorimotor cortices. ${ }^{14,48}$ Albeit the present data suggest normal sensorimotor plasticity and altered M1 inhibition in SD, they do not exclude that other nodes of the network might play a role. For instance, the cerebellum appears to have a crucial function in this context. ${ }^{49}$ The integrity of the olivo-cerebellar circuit was previously investigated in SD using an associative learning paradigm called eyeblink classic conditioning (EBCC) ${ }^{50}$ with normal results. However, this form of learning represents only part of the computation usually performed by the cerebellum, and a normal EBCC might not necessarily reflect a normal cerebellar physiology from a more general perspective. ${ }^{51} \mathrm{We}$ assume that abnormalities in the computation underlying other cerebellar functions (such as regulation of corticomotor excitability and grip force, sensorimotor synchronization, and control of movement-related sensory data acquisition ${ }^{52}$ ) might be relevant to dystonia; however, it is not known to what extent the cerebellar circuits mediating different aspects of motor control, including the EBCC, overlap. Therefore, we believe that a cerebellar dysfunction in dystonia cannot be excluded only on the basis of an intact EBCC, but this might be the object for future research. 


\section{References}

1. Albanese A, Bhatia K, Bressman SB, et al. Phenomenology and classification of dystonia: a consensus update. Mov Disord 2013;28(7): 863-873.

2. Jinnah HA, Alterman R, Klein C, et al. Deep brain stimulation for dystonia: a novel perspective on the value of genetic testing. Journal of neural transmission 2017;124(4):417-430.

3. Dressler D, Altenmueller E, Bhidayasiri R, et al. Strategies for treatment of dystonia. Journal of neural transmission 2016;123(3):251-258.

4. van den Heuvel C, Tijssen MAJ, van de Warrenburg BPC, Delnooz CCS. The symptomatic treatment of acquired Dystonia: a systematic review. Mov Disord Clin Pract 2016;3(6):548-558.

5. Neychev VK, Gross RE, Lehericy S, Hess EJ, Jinnah HA. The functional neuroanatomy of dystonia. Neurobiol Dis 2011;42(2):185-201.

6. Quartarone A, Ruge D. How many types of Dystonia? Pathophysiological Considerations. Front Neurol 2018;9:12.

7. Ridding MC, Sheean G, Rothwell JC, Inzelberg R, Kujirai T. Changes in the balance between motor cortical excitation and inhibition in focal, task specific dystonia. J Neurol Neurosurg Psychiatry 1995;59(5):493-498.

8. Huang YZ, Edwards MJ, Bhatia KP, Rothwell JC. One-Hz repetitive transcranial magnetic stimulation of the premotor cortex alters reciprocal inhibition in DYT1 dystonia. Mov Disord 2004;19(1):54-59.

9. Edwards MJ, Huang YZ, Wood NW, Rothwell JC, Bhatia KP. Different patterns of electrophysiological deficits in manifesting and non-manifesting carriers of the DYT1 gene mutation. Brain 2003; 126(Pt 9):2074-2080.

10. Kojovic M, Parees I, Kassavetis P, et al. Secondary and primary dystonia: pathophysiological differences. Brain 2013;136(Pt 7):2038-2049.

11. Espay AJ, Morgante F, Purzner J, Gunraj CA, Lang AE, Chen R. Cortical and spinal abnormalities in psychogenic dystonia. Ann Neurol 2006;59(5):825-834.

12. Quartarone A, Rizzo V, Terranova C, et al. Abnormal sensorimotor plasticity in organic but not in psychogenic dystonia. Brain 2009; 132(Pt 10):2871-2877.

13. Edwards MJ, Huang YZ, Mir P, Rothwell JC, Bhatia KP. Abnormalities in motor cortical plasticity differentiate manifesting and nonmanifesting DYT1 carriers. Mov Disord 2006;21(12): 2181-2186.

14. Conte A, Rocchi L, Latorre A, Belvisi D, Rothwell JC, Berardelli A. Ten-year reflections on the neurophysiological abnormalities of focal Dystonias in humans. Mov Disord 2019;34(11):1616-1628.

15. Conte A, Rocchi L, Ferrazzano G, et al. Primary somatosensory cortical plasticity and tactile temporal discrimination in focal hand dystonia. Clin Neurophysiol 2014;125(3):537-543.

16. Rocchi L, Erro R, Antelmi E, et al. High frequency somatosensory stimulation increases sensori-motor inhibition and leads to perceptual improvement in healthy subjects. Clin Neurophysiol 2017;128 (6):1015-1025.

17. Rocchi L, Casula E, Tocco P, Berardelli A, Rothwell J. Somatosensory temporal discrimination threshold involves inhibitory mechanisms in the primary somatosensory area. The Journal of neuroscience: the official journal of the Society for Neuroscience 2016;36(2):325-335.

18. Antelmi E, Erro R, Rocchi L, et al. Neurophysiological correlates of abnormal somatosensory temporal discrimination in dystonia. Mov Disord 2017;32(1):141-148.

19. Tinazzi M, Priori A, Bertolasi L, Frasson E, Mauguiere F, Fiaschi A. Abnormal central integration of a dual somatosensory input in dystonia. Evidence for sensory overflow. Brain 2000;123(Pt 1):42-50.

20. Erro R, Rocchi L, Antelmi, E, et al. High frequency somatosensory stimulation in dystonia: evidence fordefective inhibitory plasticity. Mov Disord 2018;33:1902-1909.

21. Comella CL, Leurgans S, Wuu J, Stebbins GT, Chmura T, Dystonia Study G. Rating scales for dystonia: a multicenter assessment. Mov Disord 2003;18(3):303-312.
22. Erro R, Rocchi L, Antelmi E, et al. High frequency repetitive sensory stimulation improves temporal discrimination in healthy subjects. Clin Neurophysiol 2016;127(1):817-820.

23. Tamura $\mathrm{Y}$, Matsuhashi $\mathrm{M}$, Lin $\mathrm{P}$, et al. Impaired intracortical inhibition in the primary somatosensory cortex in focal hand dystonia. Mov Disord 2008;23(4):558-565.

24. Leodori G, Formica A, Zhu X, et al. The third-stimulus temporal discrimination threshold: focusing on the temporal processing of sensory input within primary somatosensory cortex. J Neurophysiol 2017;118(4):2311-2317.

25. Conte A, McGovern EM, Narasimham S, et al. Temporal discrimination: mechanisms and relevance to adult-onset Dystonia. Front Neurol 2017;8:625.

26. Govert F, Becktepe J, Balint B, et al. Temporal discrimination is altered in patients with isolated asymmetric and jerky upper limb tremor. Mov Disord 2020;35(2):306-315.

27. Conte A, Ferrazzano G, Belvisi D, et al. Somatosensory temporal discrimination in Parkinson's disease, dystonia and essential tremor: pathophysiological and clinical implications. Clin Neurophysiol 2018;129(9):1849-1853.

28. Bradley $\mathrm{D}$, Whelan $\mathrm{R}$, Walsh $\mathrm{R}$, et al. Temporal discrimination threshold: VBM evidence for an endophenotype in adult onset primary torsion dystonia. Brain 2009;132(Pt 9):2327-2335.

29. Hutchinson M, Kimmich O, Molloy A, et al. The endophenotype and the phenotype: temporal discrimination and adult-onset dystonia. Mov Disord 2013;28(13):1766-1774.

30. Scontrini A, Conte A, Defazio G, et al. Somatosensory temporal discrimination in patients with primary focal dystonia. J Neurol Neurosurg Psychiatry 2009;80(12):1315-1319.

31. Kimmich O, Molloy A, Whelan R, et al. Temporal discrimination, a cervical dystonia endophenotype: penetrance and functional correlates. Mov Disord 2014;29(6):804-811.

32. Bradley D, Whelan R, Kimmich O, et al. Temporal discrimination thresholds in adult-onset primary torsion dystonia: an analysis by task type and by dystonia phenotype. J Neurol 2012;259(1):77-82.

33. Conte A, Defazio G, Hallett M, Fabbrini G, Berardelli A. The role of sensory information in the pathophysiology of focal dystonias. Nat Rev Neurol 2019;15(4):224-233.

34. Liepert J, Gorsler A, van Eimeren T, Munchau A, Weiller C. Motor excitability in a patient with a somatosensory cortex lesion. Clin Neurophysiol 2003;114(6):1003-1008.

35. Tamburin S, Zanette G. Abnormalities of sensory processing and sensorimotor interactions in secondary dystonia: a neurophysiological study in two patients. Mov Disord 2005;20(3):354-360.

36. Trompetto C, Avanzino L, Marinelli L, et al. Corticospinal excitability in patients with secondary dystonia due to focal lesions of the basal ganglia and thalamus. Clin Neurophysiol 2012;123(4): 808-814.

37. Latorre A, Rocchi L, Berardelli A, Bhatia KP, Rothwell JC. The interindividual variability of transcranial magnetic stimulation effects: implications for diagnostic use in movement disorders. Mov Disord 2019;34(7):936-949.

38. Orth M, Rothwell JC. Motor cortex excitability and comorbidity in Gilles de la Tourette syndrome. J Neurol Neurosurg Psychiatry 2009;80(1):29-34.

39. Ridding MC, Inzelberg R, Rothwell JC. Changes in excitability of motor cortical circuitry in patients with Parkinson's disease. Ann Neurol 1995;37(2):181-188.

40. Belvisi D, Suppa A, Marsili L, et al. Abnormal experimentally- and behaviorally-induced LTP-like plasticity in focal hand dystonia. Exp Neurol 2013;240:64-74.

41. Quartarone A, Morgante F, Sant'angelo A, et al. Abnormal plasticity of sensorimotor circuits extends beyond the affected body part in focal dystonia. J Neurol Neurosurg Psychiatry 2008;79(9):985-990.

42. Defazio G, Ercoli T, Erro R, et al. Idiopathic non-task-specific upper limb Dystonia, a neglected form of Dystonia, Mov Disord. 2020;35: 2038-2045.

43. Pastor MA, Day BL, Macaluso E, Friston KJ, Frackowiak RS. The functional neuroanatomy of temporal discrimination. J Neurosci 2004;24(10):2585-2591. 
44. Martino D, Liuzzi D, Macerollo A, Aniello MS, Livrea P, Defazio G. The phenomenology of the geste antagoniste in primary blepharospasm and cervical dystonia. Mov Disord 2010;25(4): 407-412.

45. Dagostino S, Ercoli T, Gigante AF, Pellicciari R, Fadda L, Defazio G. Sensory trick in upper limb dystonia. Parkinsonism Relat Disord 2019;63:221-223.

46. Svetel M, Ivanovic N, Marinkovic J, Jovic J, Dragasevic N, Kostic VS. Characteristics of dystonic movements in primary and symptomatic dystonias. J Neurol Neurosurg Psychiatry 2004;75(2): 329-330.

47. Ramos VF, Karp BI, Hallett M. Tricks in dystonia: ordering the complexity. J Neurol Neurosurg Psychiatry 2014;85(9):987-993.

48. Jinnah HA, Neychev V, Hess EJ. The anatomical basis for Dystonia: the motor network model. Tremor Other Hyperkinet Mov (N Y) 2017;7:506.

49. Latorre A, Rocchi L, Bhatia KP. Delineating the electrophysiological signature of dystonia. Exp Brain Res 2020;238(7-8):1685-1692.
50. Antelmi E, Di Stasio F, Rocchi L, et al. Impaired eye blink classical conditioning distinguishes dystonic patients with and without tremor. Parkinsonism Relat Disord 2016;31:23-27.

51. Sadnicka A, Teo JT, Kojovic M, et al. All in the blink of an eye: new insight into cerebellar and brainstem function in DYT1 and DYT6 dystonia. Eur J Neurol 2015;22(5):762-767.

52. Manto M, Bower JM, Conforto AB, et al. Consensus paper: roles of the cerebellum in motor control-the diversity of ideas on cerebellar involvement in movement. Cerebellum 2012;11(2): 457-487.

\section{Supporting Data}

Additional Supporting Information may be found in the online version of this article at the publisher's web-site. 


\section{SGML and CITI Use Only DO NOT PRINT}

\section{Author Roles}

(1) Research Project: A. Conception, B. Organization, C. Execution.

(2) Statistical Analysis: A. Design, B. Execution, C. Review and Critique.

(3) Manuscript: A. Writing of the first draft, B. Review and Critique.

Anna Latorre: 1A, 1B, 1C; 2A, 2B; 3A.

Antoniangela Cocco: 1B, 1C; 3B.

Kailash P. Bhatia: 1A, 1B; 3B.

Roberto Erro: 1B, 1C; 3B.

Elena Antelmi: 1B, 1C; 3B.

Antonella Conte: 3B.

John C. Rothwell: 2C; 3B.

Lorenzo Rocchi: 1A, 1B, 1C; 2A, 2B; 3B.

\section{Financial Disclosures}

K.P.B. has received grant support from Horizon 2020 EU grant 634,821 and honoraria/financial support to speak/ attend meetings from GSK, Boehringer-Ingelheim, Ipsen, Merz, Sun Pharma, Allergan, Teva, Lundbeck, and Orion pharmaceutical companies. K.P.B. also receives royalties from Oxford University Press and a stipend for movement disorders clinical practice (MDCP) editorship. This research study was supported by the National Institute for Health Research University College London Hospitals Biomedical Research Centre and the Edmond J. Safra Philanthropic Foundation. All the other authors have no disclosures. The authors declare that there are no conflicts of interest relevant to this work. We confirm that we have read the Journal's position on issues involved in ethical publication and affirm that this work is consistent with those guidelines. 